Sydney NSW 2052, Australia; '2Sydney Sexual Health Centre, Sydney Hospital, Sydney NSW 2000, Australia; ${ }^{3}$ School of Public Health and Community Medicine, University of New South UK, Sydney NSW 2052, Australia; " Western Sydney Sexual Health Centre, Western Sydney Local Health District, NSW, Australia; ${ }^{5}$ Sydney Emerging Infections and Biosecurity Institute, University of Sydney, NSW, Australia; ${ }^{6}$ North Shore Sexual Health Service, Royal North Shore Hospital, St Leonards NSW 2065, Australia; 'The Albion Street Centre, Surry Hills NSW 2010, Australia; ${ }^{8}$ St Vincent's Centre for Applied Medical Research, University of New South UK, Sydney NSW 2052, Australia; ${ }^{9}$ NSW State Reference Laboratory for HIV, St Vincent's Hospital, Darlinghurst NSW 2010, Australia; ${ }^{10}$ National Centre for HIV Social Research, University of New South UK, Sydney NSW 2052, Australia

Background Rapid HIV testing is well established in many countries, yet few studies have evaluated the acceptability of rapid testing among clinical staff over time. We assessed staff acceptability of rapid HIV testing before and after its implementation in Australian sexual health clinics.

Methods From September 2011 onwards, men who have sex with men (MSM) attending four Sydney sexual health clinics were offered rapid HIV testing using the Alere Determine HIV Combo assay. Staff were trained in rapid HIV testing using this assay, with staff acceptability assessed via two anonymous questionnaires completed after training and at least six months later. Five-point Likert scales were used, with ' 1 ' indicating strong agreement and ' 5 ' strong disagreement to a range of acceptability statements. T-tests were used to assess differences in mean Likert scores between rounds, with stratification by staff profession and testing experience.

Results Of 68 trained staff, 67 completed the first questionnaire and 53 the second. Mean scores improved for confidence in conducting rapid testing ( 1.87 vs $1.44 ; p<0.01)$, confidence in delivery of negative results $(1.52$ vs $1.25 ; \mathrm{p}<0.01)$ and in disagreement that rapid testing was disruptive (3.27 vs 3.83; $\mathrm{p}<0.01$ ). Comfort with your own role in rapid testing increased between rounds, particularly for nurses (1.71 vs $1.41 ; \mathrm{p}=0.04)$. In round two, doctors had a stronger preference for faster rapid tests than nurses (1.75 vs 2.50; $\mathrm{p}=0.02)$ and stronger agreement that rapid testing interferes with consultations ( 2.63 vs 3.39 ; $p=0.01$ ). Belief that patients were satisfied with rapid testing was stronger in staff who had performed $>10$ tests than $\leq 10$ tests $(1.58$ vs $2.07 ; p<0.01)$. Acceptability did not vary with experience of false results.

Conclusions Acceptability to staff of rapid HIV testing for MSM increased with time and experience of rapid testing. Differences between professions may indicate variations in staff training and support needs and capacity to adapt to change.

\section{P5.038 DEVELOPMENT OF PREDICTION RULES FOR CHLAMYDIA TRACHOMATIS INFECTION ON POPULATION AND INDIVIDUAL LEVEL - POTENTIAL FOR INNOVATIVE SELECTIVE SCREENING}

doi:10.1136/sextrans-2013-051184.1082

'D van Klaveren, ${ }^{2}, 1 \mathrm{H}$ M Götz, ${ }^{3} \mathrm{E}$ L M Op de Coul, ${ }^{1} \mathrm{E}$ W Steyerberg, ${ }^{1} Y$ Vergouwe. 'Erasmus MC, Department of Public Health, Rotterdam, The Netherlands; ${ }^{2}$ Department of Infectious Diseases, Public Health Service Rotterdam-Rijnmond, Rotterdam, The Netherlands; ${ }^{3}$ Epidemiology and Surveillance Unit, Centre for Infectious Disease Control, National Institute of Public Health and the Environment, Bilthoven, The Netherlands

Background Prediction of Chlamydia trachomatis (Ct) infection is important to identify subjects at high risk. We aimed to develop $\mathrm{Ct}$ prediction rules with different levels of detail in information, i.e. with readily available registries only and with additional detailed questionnaires.

Methods In the Chlamydia Screening Implementation (CSI) study all inhabitants of Rotterdam and Amsterdam aged 16-29 were invited yearly from 2008 until 2011 for home based urine testing. This resulted in 80,385 unique participants of whom 3,440 were infected. In addition to registry data (gender, age, ethnicity, neighbourhood level socioeconomic status [SES]) participants were asked to fill in a questionnaire on education, STI history, symptoms, partner information and sexual behaviour. We developed prediction rules based on registry risk factors only and with additional questionnaire risk factors by multilevel logistic regression to account for clustering within neighbourhoods. We assessed the discriminative ability by the area under the receiver operating characteristic curve (AUC). The models were internally validated with a bootstrap procedure.

Results Strong registry based predictors for Ct infection were young age (especially for women) and either Surinamese, Antillean or Sub-Sahara-African ethnicity. SES was of minor importance especially when questionnaire predictors were added. From the questionnaire, low to intermediate education level, ethnicity of the partner (non-Dutch) and having sex with casual partners showed strong associations with $\mathrm{Ct}$ infection. The AUC at internal validation was 0.67 based on registry risk factors only and 0.75 when questionnaire risk factors were added. To find $80 \%$ of the Chlamydia infections approximately $50 \%$ of the population needed to be screened when using the prediction rule including questionnaire risk factors.

Conclusion The registry based prediction rule can potentially facilitate selective $\mathrm{Ct}$ screening at population level, with further refinement at the individual level by including questionnaire risk factors.

\section{P5.039 TITLE: CAN STD CLINICS RIDE THE CERVICAL CANCER SCREENING BIKE? EXPERIENCES FROM AN URBAN STD CLINIC}

doi:10.1136/sextrans-2013-051184.1083

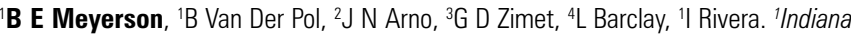
University School of Public Health-Bloomington, Bloomington, IN, United States; ${ }^{2} B e l l$ Flower Clinic, Marion County Health and Hospitals Corporation, Indianapolis, IN, United States; 'Indiana University School of Medicine, Indianapolis, IN, United States; ${ }^{4}$ American Sexual Health Association, Durham, NC, United States

Identifying screening venues that reach underserved women may be an important step in reducing cervical cancer. STD clinics often serve women who may not have ready access to other healthcare services. An urban US STD clinic was selected to test suitability of the STD clinic setting for cervical cancer screening.

Methods Women 30 years of age and older were recruited from clinic waiting rooms and compensated $\$ 20$ for study participation. Women were offered cervical cancer screening and were asked to be available for follow up by a method of their choosing. Liquid based cytology and HPV DNA (Roche, Indianapolis, IN) testing were both performed. HPV DNA assays were performed comparing results of vaginal self- swab specimens to cervical swab results. Up to 3 attempts were made to contact participants with results using the patient-preferred contact method. Outcomes of interest included reasons for not accepting screening and proportion of results delivered to this classically hard-to-reach population.

Findings At the time of this abstract, 52 participants have completed follow up (age range 30-48, median age of 37 years); 30 African American women, 19 White, 1 Native American and 2 of multiple races). Only 2 eligible clinic patients refused study participation, 47 were available for follow up contact, and 35 reported having no regular doctor. Sixteen were high risk HPV positive and 4 of this group had an accompanying pap result of LSIL or greater.

Conclusion The findings suggest that cervical cancer screening can be successfully done in this urban STD clinic setting. Despite concerns regarding the willingness of this population to provide reliable contact information, our experience suggests that women are eager to receive cervical cancer screening results. Future studies will evaluate staff provision of follow up and navigation services in a variety of STD clinic settings. 